
Cahiers
de ta Recherche
For les Droits
Fondamentaux
Cahiers de la recherche sur les droits fondamentaux

5 | 2006

L'enfant

\title{
Les réponses du droit international à la question des enfants soldats
}

Jean-Manuel Larralde

\section{(2) OpenEdition}

1 Journals

Édition électronique

URL : https://journals.openedition.org/crdf/7177

DOI : $10.4000 /$ crdf.7177

ISSN : 2264-1246

Éditeur

Presses universitaires de Caen

Édition imprimée

Date de publication : 31 décembre 2006

Pagination : 65-78

ISBN : 978-2-84133-277-9

ISSN : $1634-8842$

Référence électronique

Jean-Manuel Larralde, "Les réponses du droit international à la question des enfants soldats ", Cahiers de la recherche sur les droits fondamentaux [En ligne], 5 | 2006, mis en ligne le 15 décembre 2020, consulté le 14 novembre 2022. URL : http://journals.openedition.org/crdf/7177 ; DOI : https:// doi.org/10.4000/crdf.7177 


\title{
Les réponses du droit international à la question des enfants soldats
}

\author{
Jean-Manuel LARRALDE \\ Maître de conférences \\ Université de Caen Basse-Normandie \\ Directeur adjoint du CRDFED
}

I. Un ensemble normatif complexe

A. La protection issue des règles de droit international humanitaire

$B$. Les règles de protection issues d'autres sources supranationales

II. Une pluralité d'acteurs internationaux impliqués dans la protection des enfants soldats

A. L'intervention d'organes de " pression " vis-à-vis des États

B. L'apparition progressive d'institutions de coercition et de répression

III. Un bilan en " demi-teinte »

A. Un droit protecteur mais insuffisamment efficient

B. Un difficile accompagnement du retour à la vie civile des enfants soldats

Si lors des guerres, les civils ont toujours constitué une population particulièrement vulnérable, l'après-guerre froide a vu se multiplier des conflits souvent déclenchés pour l'exploitation et la maitrise des ressources naturelles, qui ont généré des troubles prolongés et des violences et maltraitances multiples infligées aux enfants. Le nombre d'enfants touchés directement ou indirectement par les conflits armés est considérable: selon l'UNICEF, au cours de la seule décennie 1990-2000, 2 millions d'enfants ont été tués et 6 millions mutilés ou gravement blessés; environ 10 millions d'enfants sont réfugiés ou déplacés ${ }^{1}$.

Les enfants ne sont plus seulement les victimes des conflits, ils en deviennent de plus en plus des acteurs, en raison du développement du phénomène des «enfants soldats ». S'ils ne font l'objet d'aucune définition juridique, on peut toutefois s'accorder pour dire que ce sont des enfants - garçons ou filles - de moins de 18 ans, intégrés à une force armée ou à un groupe armé régulier ou irrégulier quelconque, pour y remplir des fonctions de tous types, y compris, mais non exclusivement celles de cuisinier, porteur, planton, et toute personne accompagnant les groupes de ce type à l'exclusion des parents proches, ce qui inclut les filles et les garçons recrutés aux fins de rapports sexuels forcés et / ou de mariage forcé. La définition, large, ne s'applique donc pas uniquement à un enfant qui porte ou a porté des armes².

1. Voir «L'UNICEF dans les situations d'urgence», http://www.unicef.org/frenc/emerg/index_33296.html.

2. Définition connue sous le nom de «principes du Cap » : principes et meilleures pratiques pour la prévention du recrutement des enfants dans les forces armées et la démobilisation et la réintégration sociale des enfants soldats en Afrique, symposium du Cap du 30 avril 1997. Adoptés à l'issue d'une conférence internationale sur les enfants soldats, ces principes ont été acceptés par un grand nombre d'organismes de protection de l'enfant, d'organisations non gouvernementales et d'organismes des Nations Unies, parmi lesquels l'UNICEF et la Banque mondiale. 
L'existence d'enfants soldats est aujourd'hui loin d'être marginale, même si les chiffres avancés ne constituent que des estimations. Dans son rapport pour 1998, le représentant spécial du Secrétaire général chargé d'étudier l'impact des conflits armés sur les enfants indiquait que 250000 mineurs de moins de 18 ans combattaient dans des conflits en cours ${ }^{3}$. Dans un entretien plus récent, ce même représentant spécial estimait ce nombre à 300000 , en particulier parce que les conflits sont de plus en plus des guerres civiles ${ }^{4}$. Des sources officielles évoquent la présence de plus de 30000 enfants soldats en République démocratique du Congo ${ }^{5}$, le même chiffre étant avancé pour le recrutement d'enfants au sud Soudan par l'armée de résistance du Seigneur (LRA) entre 1986 et $2006^{6}$. La prise de Mogadiscio en juin 2006 par les forces islamistes a récemment montré l'utilisation continue d'enfants par les différentes factions au conflit, ce qui constitue désormais une pratique bien ancrée en Somalie. Même si toutes les parties du globe sont aujourd'hui concernées par la question, la présence d'enfants soldats est particulièrement forte en Birmanie, en Colombie, en Côte d'Ivoire, dans certaines régions de la République du Congo, au Liberia et dans le nord de l'Ouganda.

Des enfants, de plus en plus jeunes ${ }^{7}$, sont entraînés en nombre croissant dans des combats dont les enjeux les dépassent très largement. Cette catégorie de population, qui devrait normalement être préservée des actions armées, se trouve ainsi confrontée à de grandes souffrances (traumatismes lourds, blessures, captivité ou décès). Le taux de mortalité de ces enfants est souvent élevé, en raison de leur manque d'expérience et d'entraînement, et du fait qu'ils sont utilisés pour des missions particulièrement périlleuses, comme le renseignement ou la pose de mines antipersonnel. Les enfants ayant pris part aux hostilités souffrent beaucoup plus que les adultes, tant sur le plan psychologique que sur le plan physique et présentent des déséquilibres d'ordre psychologique ou psychique souvent graves, à une période de la vie, l'adolescence, où chaque individu incorpore normes et valeurs sociales.

Il s'agit là d'une source importante de violations du droit humanitaire. En effet, les enfants peuvent aisément être incités (ou forcés) à perpétrer des actes dont ils ne mesurent nullement ni l'exacte portée, ni la gravité. La situation des mineurs combattants est, en outre, très complexe à prendre en compte, en raison des particularismes des champs de bataille. Ainsi, dans certaines hypothèses, les jeunes sont choisis comme recrues et exploités comme combattants à la fois par les forces armées gouvernementales ${ }^{8}$ et par les groupes armés d'opposition 9 .

La complexité de la question des enfants soldats est renforcée par les causes de développement de ce phénomène, multiples et parfois combinées. Pour la plupart des commentateurs, leur apparition correspond d'abord à une transformation de la nature des conflits: jusqu'à la Seconde Guerre mondiale, les conflits opposaient surtout des armées régulières. L'utilisation massive d'enfants soldats renvoie à des conflits opposant armées régulières et guérilla ${ }^{10}$. Comme le souligne Olara Otunnu, l'ancien représentant spécial des Nations Unies pour les enfants et les conflits armés, la Première Guerre mondiale «était une guerre de soldat à soldat, avec peu de victimes civiles, $5 \%$ tout au plus. La Seconde Guerre mondiale a été terrible, surtout vers la fin, à l'époque des grands bombardements : $45 \%$ des victimes étaient des civils, blessés, tués, déplacés. Aujourd'hui, ce sont des "guerres à l'envers", soldats contre civils, des guerres modernes qui touchent et emportent femmes et enfants $" ~ "$. Mais cette raison est loin d'être unique et le développement des enfants combattants correspond à d'autres logiques ${ }^{12}$.

Lors de guerres de longue durée ( 40 ans de conflit en Colombie, 25 ans en Angola, plus de 20 ans en Afghanistan...) le recrutement d'adultes devient de plus en plus difficile. Le recrutement des enfants s'effectue alors pour compenser l'hécatombe des vétérans ou corriger le déséquilibre initial des forces. Les enfants combattants sont souvent placés dans les groupements supplétifs où ils risquent moins de susciter, par leur inexpérience et leur indiscipline, l'agacement des combattants de métier. Les enfants peuvent également être affectés à de multiples tâches, car s'ils savent vite se battre, tuer, ils servent également aux cuisines, portent les messages, s'infiltrent, jouent les agents de renseignement. Les enfants, et surtout les

3. Document E/CN.4/1998/119.

4. «Enfants-soldats: cessons d'aider ceux qui les arment! Un entretien avec le Secrétaire général adjoint des Nations unies», Le Nouvel Observateur, 25 mai 2000, $\mathrm{n}^{\circ}$ 1855. Le même chiffre est repris récemment dans le ICRC Annual Report 2005, Genève, mai 2006.

5. Doc. E / CN.4/ $2003 / 43, \$ 33$ à 36 .

6. Voir P. Bernard, «Enfances volées en Ouganda », Le Monde, 22 février 2006.

7. On trouve mention du recrutement d'enfants de 5 ans en Sierra Leone, et de l'enrôlement forcé d'enfants de 11 ans par l'armée nationale birmane. Voir respectivement Comité des droits de l'enfant, Observations finales: Sierra Leone, doc. CRC / C/15/ Add.116 24 février 2000, $\$ 71 ;$ Rapport du Secrétaire général sur les enfants et les conflits armés, doc. S/ 2003/1053, S.

8. La Coalition pour mettre fin à l'utilisation d'enfants soldats indiquait en 2003 que les enfants représentent $35 \%$ à $45 \%$ des nouvelles recrues de l'armée nationale et quelque 70000 au moins des soldats birmans estimés à 350000 . Les enfants soldats sont également utilisés par les armées appartenant aux minorités ethniques. Voir http://www.child-soldiers.org; doc. S/ $2003 / 1053$, S.

9. Voir Droits de l'enfant: l'avenir en dépend, Paris, Amnesty International, 1999, p. 5.

10. Même si l'on peut souligner des exemples historiques, tels que l'utilisation des Hitlerjugend pendant la Deuxième Guerre mondiale. À partir de 1943, les chefs nazis firent des Jeunesses hitlériennes une réserve militaire (ainsi la $12^{\text {e }}$ division SS était entièrement composée de jeunes garçons entre 16 et 18 ans). En 1945, la Volkssturm mobilisait même de jeunes enfants de 10 ans.

11. Le Nouvel Observateur, 25 mai 2000.

12. Concernant les causes de l'enrôlement des enfants et les modalités de leur utilisation, voir notamment le rapport de G. Machel, L'Impact des conflits armés sur les enfants, doc. A / 51/306, 26 août 1996. 
jeunes filles, sont les « instruments » préférés des organisateurs de " commandos suicides", lorsqu'ils ne sont pas violés ou utilisés comme esclaves sexuels.

Il ne faut pas non plus oublier que les enfants sont facilement impressionnables et peuvent être facilement transformés en de redoutables guerriers. Le recrutement d'enfants parmi les combattants répond alors à une véritable stratégie. En Sierra Leone, au Sri Lanka ou au Rwanda, les pires atrocités à l'encontre des populations civiles ont parfois été commises par des enfants. Comme l'indique M.-T. Dutli, «L'enfant qui participe aux hostilités est non seulement placé lui-même en danger de mort, mais également les personnes qui deviennent sa cible, à cause de son comportement immature et passionné ${ }^{13}$. Pour un chef de guerre, un enfant est un « outil» plus malléable, qui ne se rend pas toujours totalement compte de ses actes, facile à manipuler et à façonner. L'enfant devient ainsi une menace pour l'ensemble de la population civile. Pour améliorer leurs performances militaires, ces enfants sont souvent drogués avant les combats, ce qui s'ajoute à l'endoctrinement, aux contraintes et menaces ${ }^{14}$. Afin de mieux s'assurer de la population, on leur ordonne parfois de terroriser, de torturer ou de massacrer leur propre famille dans le village où ils ont grandi. Coupables et salis, maudits par leurs parents et exclus de leur communauté, ces enfants deviennent des parias, condamnés à rester avec leur groupe armé ${ }^{15}$.

En outre, les enfants peuvent également rejoindre les combattants parce que leurs conditions de vie ne leur offrent aucune autre alternative viable. Ainsi en Irlande du Nord, l'enrôlement et l'utilisation d'enfants par les groupes paramilitaires ont fortement augmenté dans les années 1970, en raison de la persistance de la peur, de perceptions sectaires, mais aussi de facteurs socio-économiques défavorables. Cette situation est encore plus compréhensible lorsque l'enfant ne dispose d'aucune autre possibilité de survie: dans un pays qui s'est effondré, avec des écoles en ruine, l'absence de travail, une famille disparue, il ne reste parfois plus que la perspective de rejoindre une unité de combattants, qui offre un uniforme pour se vêtir, de quoi manger... La prise en compte des avantages financiers constitue l'une des explications fréquentes de l'enrôlement. L'enfant est même alors souvent encouragé par ses parents, qui parfois n'ont pas les moyens d'assurer l'entretien de la famille.

Dans d'autres cas, les enfants sont endoctrinés par des appels idéologiques, religieux, ou politiques. Il s'agit d'ailleurs de cas où l'engagement de l'enfant peut parfois être considéré comme réellement volontaire. Ainsi au Sri Lanka ou en Colombie, où le contexte idéologique et patriotique est très fort, de nombreuses jeunes filles sont plutôt volontaires, fuyant la pauvreté, mais trouvant aussi dans l'engagement militaire une reconnaissance sociale qu'elles pensent trouver au combat car elles sont égales à l'homme. Dans toutes ces hypothèses renvoyant au «volontariat » de l'enfant, il est toujours indispensable de distinguer entre ces cas d'engagement personnel véritable et ceux où l'enfant a été influencé, manipulé, voire endoctriné par des adultes.

Des raisons liées à la sécurité physique de l'enfant existent également souvent : les enfants témoins de meurtres ou de massacres seront plus enclins à rejoindre les forces ou groupes armés, au sein desquels ils pensent être plus en sécurité face aux dangers existants. Ainsi au Sri Lanka, les filles servent dans les troupes indépendantistes des Tigres tamouls (LTTE), car elles se sentent davantage "protégées», les relations sexuelles entre combattants étant interdites.

Enfin, la prolifération des armes légères, peu coûteuses ${ }^{16}$ et facilement utilisables par des personnes disposant de peu de force physique ou de connaissances techniques, a également permis le recours à de très jeunes enfants. La diffusion des armes AK 47 ou M 16, qui peuvent être aisément démontées par un enfant de 10 ans, a contribué à l'utilisation de jeunes combattants.

Face à ce phénomène particulièrement complexe, la communauté internationale n'est pas restée inactive. En février 2000, M. Olara Otunnu, représentant spécial du Secrétaire général des Nations Unies pour les enfants et les conflits armés, présentait de manière synthétique les éléments que l'on peut considérer comme relevant de la politique menée par les Nations Unies en vue d'éliminer l'enrôlement d'enfants lors des conflits : préconiser expressément que 18 ans soit l'âge minimum de la participation aux conflits; surveiller le respect, par toutes les parties aux conflits, des engagements qu'elles ont conclus en matière de protection des enfants, et rendant ce respect obligatoire; exercer une pression internationale sur les parties belligérantes qui maltraitent les enfants en s'en servant comme combattants; s'attaquer aux facteurs politiques, sociaux et économiques qui facilitent l'exploitation des enfants comme soldats; répondre aux besoins des exenfants soldats en matière de réinsertion sociale; et élargir le champ des interventions à tous les enfants touchés par des conflits ${ }^{17}$. Le droit international a effectivement pris en compte la question des enfants à un double niveau :

13. Voir notamment M.-T. Dutli, «Enfants-combattants prisonniers», Revue internationale de la Croix-Rouge, $\mathrm{n}^{\circ} 785$, septembre-octobre 1990 , p. 456 sq.

14. En Sierra Leone, le recrutement effectué par les Kamajors illustre cette forme d'initiation à la violence. Ces chasseurs traditionnels et professionnels ont souvent recours à la magie. Presque toujours recouverts d'amulettes, de gris-gris, de griffes et de poils d'animaux, ce qui forme en quelque sorte leur bouclier de combat, ces Kamajors initient les enfants en leur faisant ingurgiter une boule de viande composée notamment de chair humaine et boire une calebassée de sang humain, ingrédients qui rendent féroce, cruel et protègent contre les balles sifflantes. Les enfants sont facilement convaincus des bienfaits des objets des Kamajors et ils se laissent ainsi emporter dans l'initiation pour pouvoir prendre part aux hostilités (C. Charlot, Les Enfants soldats en Sierra Leone, Mémoire de DEA de relations internationales, Université Panthéon-Assas - Paris II, 2001, p. 14-15).

15. Le Nouvel Observateur, 25 mai 2000.

16. Dans son rapport de 1996, G. Machel indique qu'en Ouganda, un fusil automatique AK 47 peut être acheté pour le prix d'un poulet et dans le nord du Kenya, le coût est équivalent à celui d'une chèvre (L'Impact des conflits armés sur les enfants, \$27).

17. Doc E/CN.4/ 2000 / 71, 9 février 2000. 
un encadrement normatif complexe tend à renforcer la prohibition du recours aux enfants soldats (I). Cette progression du droit s'accompagne d'interventions de multiples acteurs institutionnels (II). Malgré ce double engagement, les résultats restent fragiles et partiels, tout spécialement dans le domaine de la démobilisation des enfants soldats et de leur retour à la vie civile (III).

\section{Un ensemble normatif complexe}

Parce que le phénomène de l'exploitation des enfants en tant que soldats est de plus en plus généralisé, la communauté internationale a élaboré de nombreux instruments destinés à les protéger et à les prémunir contre une implication dans les conflits armés. Ces instruments renvoient en partie aux règles de droit humanitaire issues du "droit de Genève » ${ }^{18}(\mathrm{~A})$, mais émanent de plus en plus d'autres sources, rendant à la fois la construction normative plus protectrice et plus complexe à mettre en œuvre (B).

\section{A. La protection issue des règles de droit international humanitaire}

L'appellation « enfant soldat » ne correspond à aucun statut juridique spécifique ${ }^{19}$ et pour le droit international et le droit international humanitaire, l'enfant en période de conflit est avant tout une personne vulnérable, qui doit faire l'objet de protections renforcées ${ }^{20}$. Par contre, pour ce même droit humanitaire, les enfants membres des forces armées ont le statut de combattant, ce qui signifie clairement que lors d'un conflit armé, ils ont le droit de tuer et peuvent être tués puisqu'ils peuvent faire l'objet d'attaques, en tant que cibles licites ${ }^{21}$. L'enfant combattant (qu'il fasse ou non partie des forces armées) peut, en outre, par faitement être puni en vertu de la législation interne du pays concerné pour le seul fait d'avoir pris part aux hostilités ${ }^{22}$. L'étendue de sa responsabilité devrait toutefois être appréciée en prenant en considération sa capacité restreinte de discernement, inhérente à son jeune âge.

Les enfants ayant pris part aux hostilités n'ont donc pas droit à un statut particulier ${ }^{23}$. Ils ne bénéficient que de la protection générale reconnue par le premier alinéa de l'article 75 du Protocole $\mathrm{I}^{24}$. Cette disposition vise toutes les personnes qui sont au pouvoir d'une partie au conflit et qui ne bénéficient pas d'un traitement plus favorable en vertu des Conventions et du Protocole. Elle énonce, en outre, un minimum de règles humanitaires reconnues en faveur de toutes les personnes affectées par un conflit armé, y compris les enfants.

La capture et l'internement des enfants soldats montrent d'ailleurs bien la volonté de faire de ceux-ci des combattants à part entière.

Les enfants, quel que soit leur âge, qui sont recrutés ou engagés volontaires dans les forces armées, ont la qualité de combattants et bénéficient en cas de capture du statut de prisonniers de guerre. Les enfants combattants capturés, de moins de 15 ans, ne pourront toutefois pas être condamnés pour avoir pris les armes. Leur participation aux hostilités n'entraîne aucune faute de leur part, étant donné que l'interdiction visée par l'article 77, paragraphe 2 du Protocole I s'adresse aux parties au conflit et non aux enfants.

Toutefois, dans les conflits armés non internationaux, il n'existe ni statut de combattant ni celui qui en découle, le statut de prisonnier de guerre. Il n'y a pas non plus de catégories de personnes civiles protégées, ni d'internés civils. Un enfant combattant capturé dans un conflit armé non international reste seulement soumis au bénéfice de la protection reconnue par l'article 3 commun aux Conventions de Genève qui s'applique à toutes les personnes qui

18. Formé des quatre Conventions du 12 août 1949 (ci-dessous « $\mathrm{I}^{\mathrm{re}}, \mathrm{II}^{\mathrm{e}}, \mathrm{III}^{\mathrm{e}}$ et $\mathrm{IV}^{\mathrm{e}}$ Conventions de Genève »), auxquelles il convient d'ajouter les deux Protocoles additionnels du 8 juin 1977, l'un relatif à la protection des victimes des conflits armés internationaux (ci-dessous «Protocole I»), l'autre relatif à la protection des victimes des conflits armés non internationaux (ci-dessous « Protocole II »). Les quatre Conventions de Genève ont été ratifiées par 192 États, les deux Protocoles par respectivement 164 et 159 États au 15 juin 2006.

19. Et il faut attendre 1977, avec la rédaction des Protocoles additionnels aux Conventions de Genève, pour qu'apparaisse pour la première fois dans un texte juridique contraignant la notion d'enfants participant aux conflits armés. L'absence de toute disposition concernant les enfants soldats dans les quatre Conventions de Genève de 1949 s'expliquerait par la considération, répandue à l'époque, que le droit international humanitaire ne devait pas s'interposer entre les États et leurs ressortissants et toucher à la souveraineté des États. Voir F. Bugnion, «Les enfants-soldats, le droit international humanitaire et la Charte africaine des droits et du bien-être de l'enfant », Revue africaine de droit international et comparé, t. $12, \mathrm{n}^{\circ}$ 2, $2000, \mathrm{p} .266$.

20. Pour l'ensemble des dispositions de droit humanitaire spécialement applicables aux enfants dans les conflits armés, voir le tableau de synthèse établi par le Comité international de la Croix-Rouge. Source: http://www.cicr.org/web/fre/sitefreo.nsf/html/5HJJ4C/\$File/FRAo3_04_tableau_DIH_TOTAL_logo. PDF.

21. Article 43 du Protocole I: « les forces armées d'une Partie à un conflit se composent de toutes les forces, tous les groupes et toutes les unités armés et organisés qui sont placés sous un commandement responsable de la conduite de ses subordonnés devant cette Partie...».

22. Le droit international humanitaire fait souvent référence à la participation aux «hostilités » et non aux " conflits armés », ce qui permet donc l'application des règles en cas de conflit armé de faible intensité.

23. En vertu de l’article $45 \$ 3$ du Protocole I.

24. «Dans la mesure où elles sont affectées par une situation visée à l'article premier du présent Protocole, les personnes qui sont au pouvoir d'une Partie au conflit et qui ne bénéficient pas d'un traitement plus favorable en vertu des Conventions et du présent Protocole seront traitées avec humanité en toutes circonstances et bénéficieront au moins des protections prévues par le présent article sans aucune distinction de caractère défavorable fondée sur la race, la couleur, le sexe, la langue, la religion ou la croyance, les opinions politiques ou autres, l'origine nationale ou sociale, la fortune, la naissance ou une autre situation, ou tout autre critère analogue. Chacune des Parties respectera la personne, l'honneur, les convictions et les pratiques religieuses de toutes ces personnes». 
ne participent pas, ou ne participent plus, aux hostilités ${ }^{25}$. Ces enfants bénéficient en outre de la protection qui leur est reconnue par l'article $77 \S 1$ du Protocole $I^{26}$ et de l'article $4 \$ 3$ du Protocole II, lequel donne des précisions sur les soins et l'aide dont doivent bénéficier tous les enfants dans un tel conflit ${ }^{27}$. Cette liste n'étant pas limitative, elle ne préjuge en rien d'autres mesures qui devraient être prises en leur faveur par les États.

Mais le droit international humanitaire procède en même temps d'une autre logique : les enfants (et tout spécialement les plus jeunes d'entre eux) n'ont pas vocation à participer aux conflits armés. La meilleure solution réside donc dans l'instauration d'une prohibition du recrutement et de l'utilisation d'enfants lors des conflits armés. Pour les conflits armés internationaux, la disposition centrale est l'article 77, Paragraphe 2 du Protocole I, qui fixe la limite à 15 ans pour le recrutement (volontaire ou sous la contrainte) et la participation «directe» des enfants aux hostilités, et qui encourage les États, en cas de recrutement de personnes entre 15 et 18 ans, à incorporer d'abord les plus âgés ${ }^{28}$. En situation de conflit armé non international, c'est l'article 4, paragraphe 3, alinéa c) du Protocole II qui se réfère de nouveau à l'âge de 15 ans, au-dessous duquel les enfants n'ont pas le droit de participer aux hostilités ${ }^{29}$. Il s'agit en l'occurrence d'une interdiction absolue, qu'elle vise une participation directe ou indirecte aux hostilités ${ }^{30}$. L'obligation imposée aux États parties est donc plus stricte que lors de conflits armés internationaux.

\section{B. Les règles de protection issues d'autres sources supranationales}

Il n'est guère étonnant que l'instrument international spécialement dédié à la protection des enfants, la Convention relative aux droits de l'enfant du 20 novembre $1989^{31}$, se prononce sur la situation des enfants en situation de conflit. Cependant, de manière relativement surprenante, cette Convention ne marque pas de progrès par rapport aux règles issues du droit humanitaire, puisqu'elle se contente de reprendre, dans son article 38 , le libellé de l'article 77, paragraphe 2 du Protocole I ${ }^{32}$. Cette disposition ${ }^{33}$ interdit ainsi la participation directe aux hostilités des enfants de moins de 15 ans. Elle est même plus faible que le droit existant dans la mesure où le droit international humanitaire applicable aux conflits armés non internationaux interdit toute participation directe et « indirecte » de ces enfants aux hostilités. L'article 38, paragraphe 1 contient toutefois une clause de renvoi aux règles du droit international humanitaire, dont la protection s'étend aux enfants $^{34}$. Il faut également relever que l'article 39 de cette Convention prévoit que «les États parties prennent toutes les mesures appropriées pour faciliter la réadaptation physique et psychologique et la réinsertion sociale de tout enfant » victime notamment de conflit armé (ce qui inclut les victimes civiles, mais aussi les enfants soldats). Cette réadaptation et cette réinsertion doivent se dérouler « dans des conditions qui favorisent la santé, le respect de soi et la dignité de l'enfant».

25. Disposition qui prévoit que ces personnes doivent être traitées «avec humanité", ce qui interdit les atteintes portées à la vie et à l'intégrité corporelle, notamment le meurtre sous toutes ses formes, les mutilations, les traitements cruels, tortures et supplices; les prises d'otages; les atteintes à la dignité des personnes, notamment les traitements humiliants et dégradants; les condamnations prononcées et les exécutions effectuées sans un jugement préalable, rendu par un tribunal régulièrement constitué, assorti des garanties judiciaires reconnues comme indispensables par les peuples civilisés. En outre, les malades et blessés doivent être recueillis et soignés.

26. «Les enfants doivent faire l'objet d'un respect particulier et doivent être protégés contre toute forme d'attentat à la pudeur. Les Parties au conflit leur apporteront les soins et l'aide dont ils ont besoin du fait de leur âge ou pour toute autre raison ».

27. «Les enfants recevront les soins et l'aide dont ils ont besoin et, notamment: a) ils devront recevoir une éducation, y compris une éducation religieuse et morale, telle que la désirent leurs parents ou, en l'absence de parents, les personnes qui en ont la garde ; b) toutes les mesures appropriées seront prises pour faciliter le regroupement des familles momentanément séparées; c) les enfants de moins de quinze ans ne devront pas être recrutés dans les forces ou groupes armés, ni autorisés à prendre part aux hostilités; d) la protection spéciale prévue par le présent article pour les enfants de moins de quinze ans leur restera applicable s'ils prennent directement part aux hostilités en dépit des dispositions de l’alinéa c et sont capturés; e) des mesures seront prises, si nécessaire et, chaque fois que ce sera possible, avec le consentement des parents ou des personnes qui en ont la garde à titre principal en vertu de la loi ou de la coutume, pour évacuer temporairement les enfants du secteur où des hostilités ont lieu vers un secteur plus sûr du pays, et pour les faire accompagner par des personnes responsables de leur sécurité et de leur bien-être ».

28. «Les Parties au conflit prendront toutes les mesures possibles dans la pratique pour que les enfants de moins de quinze ans ne participent pas directement aux hostilités, notamment en s'abstenant de les recruter dans leurs forces armées. Lorsqu'elles incorporent des personnes de plus de quinze ans mais de moins de dix-huit ans, les Parties au conflit s'efforceront de donner la priorité aux plus âgées ».

29. «Les enfants de moins de quinze ans ne devront pas être recrutés dans les forces ou groupes armés, ni autorisés à prendre part aux hostilités».

30. Cette participation indirecte pouvant consister en des actions extrêmement diverses, telle que la collecte de renseignements, la transmission d'ordres, le transport de munitions et de vivres, ou encore des actes de sabotage.

31. Entrée en vigueur le 2 septembre 1990, elle lie actuellement 192 États parties.

32. «1. Les États parties s'engagent à respecter et à faire respecter les règles du droit humanitaire international qui leur sont applicables en cas de conflit armé et dont la protection s'étend aux enfants. 2. Les États parties prennent toutes les mesures possibles dans la pratique pour veiller à ce que les personnes n'ayant pas atteint l'âge de quinze ans ne participent pas directement aux hostilités. 3. Les États parties s'abstiennent d'enrôler dans leurs forces armées toute personne n'ayant pas atteint l'âge de quinze ans. Lorsqu'ils incorporent des personnes de plus de quinze ans mais de moins de dix-huit ans, les États parties s'efforcent d'enrôler en priorité les plus âgées. »

33. Qui fut adoptée dans la plus grande confusion. Voir notamment F. Krill, «The Protection of Children in Armed Conflicts », in The Ideologies of Children's Rights, M. Freeman, P. Veerman (éd.), Leyde, Martinus Nijhoff Publishers, 1992, p. 353.

34. En raison de cette clause, ainsi que du caractère de lex specialis du droit international humanitaire, en cas de doute, c'est l'article 4, paragraphe 3, alinéa c) du Protocole II qui s'applique. Cette dernière disposition accorde à l'enfant une protection plus grande. 
La rédaction d'un protocole facultatif à la Convention relative aux droits de l'enfant, concernant l'implication d'enfants dans les conflits armés, adopté le 25 mai $2000^{35}$ a constitué une nouvelle étape décisive ${ }^{36}$, comblant les lacunes révélées par l'article 38 de la Convention de $1989^{37}$. L'article premier procède au relèvement à 18 ans de l'âge minimum autorisé pour la participation directe aux hostilités ${ }^{38}$. De manière analogue, l'article 2 relève à 18 ans l'âge du recrutement ${ }^{39}$. La protection reste toutefois relative en raison de la double dérogation contenue dans l'article 3, par laquelle les enfants d'écoles militaires, quel que soit leur âge, sont assimilés à des cibles légales et ne sont couverts que par la protection classique apportée par le droit international humanitaire à l'égard des combattants. Pour les enfants engagés volontairement, l'âge limite d'incorporation est abaissé à 15 ans dans la mesure où l'engagement réunit certaines conditions énoncées dans cet article ${ }^{40}$. Enfin, l'article 4 du Protocole facultatif constitue un apport essentiel au droit existant puisqu'il met à la charge de toutes les parties au conflit, y compris aux groupes armés distincts des forces armées officielles, l'application des dispositions prévues aux articles 1 et 2 . En particulier, les groupes armés insurgés ne doivent « en aucune circonstance » recruter ou utiliser dans les hostilités les personnes âgées de moins de 18 ans. De manière tout à fait intéressante, ces nouvelles normes ont vocation à s'appliquer aussi bien aux conflits internationaux qu'aux guerres civiles.

Si l'ensemble formé par les normes du droit de Genève et de la Convention des droits de l'enfant et de son Protocole additionnel comprend l'essentiel des références opératoires dans le domaine de la protection des enfants soldats, il convient de relever que d'autres normes contribuent à cette protection, notamment en ce qui concerne l'enrôlement des enfants.

Avec la Convention concernant l'interdiction des pires formes de travail des enfants et l'action immédiate en vue de leur élimination, adoptée à l'unanimité des 174 États membres de l'Organisation internationale du travail le 17 juin 1999, le recrutement forcé ou obligatoire des enfants en vue de leur utilisation dans les conflits armés constitue l'une des «pires formes de travail des enfants ${ }^{41}$. Cette Convention, qui s'applique à toutes les personnes de moins de 18 ans, oblige les États parties à « prendre des mesures immédiates et efficaces pour assurer l'interdiction et l'élimination» de ces pratiques ( $\left.\operatorname{art} .1^{\mathrm{er}}\right)$. La Convention préconise, en outre, l'élaboration de programmes d'action pour éliminer le recours aux enfants soldats et la prise de «toutes les mesures nécessaires pour assurer la mise en œuvre effective et le respect des dispositions..., y compris par l'établissement et l'application de sanctions pénales ou, le cas échéant, d'autres sanctions » (art. 7).

On trouve même une prise en considération régionale du phénomène des enfants soldats, tout spécialement sur le continent africain, particulièrement touché par ce fléau. La Conférence des chefs d'État et de gouvernement de l'Organisation de l'unité africaine a adopté, en juillet 1999, la Déclaration d'Alger dans laquelle les participants se sont engagés à lutter contre toutes les formes d'exploitation des enfants et, en particulier, à mettre fin au phénomène des enfants soldats. Mais c'est la Charte africaine des droits et du bien-être de l'enfant adoptée à AddisAbeba en juillet 1990 et entrée en vigueur le 29 novembre 1999, qui a été le premier traité régional à fixer à 18 ans l'âge minimal de la conscription et de la participation aux hostilités. En vertu de son article $22 \$ 2$, «Les États parties à la présente Charte prennent toutes les mesures nécessaires pour faire en sorte qu'aucun enfant ne prenne part directement aux hostilités et ils s'abstiennent, en particulier, de recruter des enfants ".

Tous ces textes constituent des avancées juridiques indéniables, mais ne sont toutefois pas exempts de critiques. En particulier parce que la terminologie utilisée est largement de nature à affaiblir la protection ${ }^{42}$.

En premier lieu, la mention de "participation directe» utilisée par le droit humanitaire affaiblit considérablement la protection de l'enfant, car le droit ne prohibe qu'un certain type de participation aux hostilités. En effet, ni le Protocole facultatif ni ses travaux préparatoires ne précisent ni ne définissent les notions de participations « directe» et « indirecte». Selon le Commentaire des Protocoles additionnels, «la participation directe aux hostilités implique un lien direct de cause à effet entre l'activité exercée et les coups qui sont portés à l'ennemi, au moment où cette activité s'exerce et là où elle s'exerce ${ }^{43}$. En d'autres termes, la participation directe se limite donc

35. Entré en vigueur le 12 février 2002, il lie aujourd'hui 107 États parties. Ci-dessous « Protocole facultatif».

36. À la demande instante du représentant spécial des Nations Unies et de l'UNICEF, ce Protocole facultatif a été inclus dans la liste des traités de base auxquels le Secrétaire général a invité vivement les États membres à adhérer lors de l'Assemblée du Millénaire (avril 200o). De même, dans une résolution du 11 avril 2002, le Parlement européen a demandé à l'Union européenne et à ses États membres d'exprimer un soutien en faveur de la ratification universelle et de la mise en œuvre du protocole facultatif.

37. T. Feraro, «Le protocole facultatif sur l'implication des enfants dans les conflits armés », Revue générale de droit international public, vol. 2, 20oo, p. 481 sq.

38. Même si le caractère contraignant de la mesure est atténué par rapport au Protocole additionnel de 1977, puisqu’il énonce simplement une obligation de moyen : «les États parties prendront toutes les mesures possibles dans la pratique».

39. Ceci permet d'aligner le droit international sur d'autres normes et notamment sur l'initiative de l'OUA, qui a fixé la majorité à 18 ans, dans la Charte africaine des droits et du bien-être de l'enfant.

40. Le caractère véritablement volontaire de l'engagement, l'avis favorable des parents ou des tuteurs légaux, l'information sur les obligations découlant d'un tel engagement et la preuve formelle que l'âge de 15 ans est bien révolu constituent les éléments définissant l'engagement volontaire.

41. Article 3: "Aux fins de la présente convention, l'expression "les pires formes de travail des enfants" comprend: a) toutes les formes d'esclavage ou pratiques analogues, telles que la vente et la traite des enfants, la servitude pour dettes et le servage ainsi que le travail forcé ou obligatoire, y compris le recrutement forcé ou obligatoire des enfants en vue de leur utilisation dans des conflits armés ».

42. Le Comité international de la Croix-Rouge a rapidement mis l'accent sur les lacunes et les faiblesses du texte du Protocole additionnel. Voir Commission des droits de l'homme, $56^{\mathrm{e}}$ session, 6 avril 2000, Genève, point 13 de l'ordre du jour ; intervention du Comité international de la Croix-Rouge.

43. Les Protocoles additionnels aux Conventions de Genève du 12 août 1949, Genève, CICR, 1977. 
aux « actes de guerre que leur nature ou leur but destinent à frapper concrètement le personnel et le matériel des forces armées adverses ${ }^{44}$. Elle ne comprend donc pas des actes comme la recherche et la transmission d'informations militaires, l'espionnage, le sabotage, le transport d'armes et de munitions, le ravitaillement, les fonctions de porteur ou d'assistant aux postes de contrôle militaire, etc. ${ }^{45}$. Or ce sont justement ces tâches qui sont le plus souvent confiées aux enfants, notamment parce qu'en raison de leur taille, ils sont moins repérables, et donc plus efficaces que les adultes. En outre, un enfant membre des forces armées ou d'un groupe armé et qui participe indirectement aux hostilités est, dans la pratique, difficilement dissociable du reste des combattants. Il n'est donc pas protégé contre une attaque de l'ennemi et court les mêmes risques que toute personne qui participe directement aux hostilités.

De même, le Protocole facultatif met en place une subtile distinction entre l'enrôlement volontaire et l'enrôlement forcé. Or, dans la pratique, il n'est pas toujours facile de mettre en ouvre cette distinction. Il peut arriver, par exemple, que des volontaires aient été contraints de s'engager parce qu'ils avaient faim, avaient besoin d'une protection physique, étaient pauvres ou cherchaient à se venger. Il peut aussi s'avérer difficile de vérifier l'âge des intéressés dans les États touchés par la guerre où n'existe pas de système fiable d'enregistrement des naissances. On peut également se demander si l'exception accordée aux écoles militaires n'affaiblit pas la portée du protocole.

Les enfants soldats sont donc concernés par un ensemble dense de règles internationales, auxquelles font écho des interventions complémentaires de plusieurs acteurs internationaux.

\section{Une pluralité d'acteurs internationaux impliqués dans la protection des enfants soldats}

Les Nations Unies ont aujourd'hui mis à contribution de nombreuses institutions et cette multiplicité d'acteurs, par le renforcement de la répression pénale internationale, dépasse même le cadre strictement onusien. Le HautCommissariat des droits de l'enfant, l'UNICEF, le Comité international de la Croix-Rouge (CICR) et un grand nombre d'organisations non gouvernementales se consacrent aujourd'hui à la protection des enfants dans les conflits. On peut ainsi plus spécifiquement mettre en avant des interventions complémentaires d'organes destinés à assurer une «pression » sur les États de la communauté inter- nationale (A), auxquels s'ajoutent de plus en plus des institutions plus coercitives et même répressives (B).

\section{A. L'intervention d'organes de "pression" vis-à-vis des États}

Les dispositions contenues dans la Convention sur les droits de l'enfant et le Protocole additionnel renvoient aux «canons» habituels de la protection mise en place par ce type de texte en droit international. Pour garantir sa mise en œuvre et son application effectives, la Convention instaure un Comité des droits de l'enfant (art. 43 et suivants), composé de dix-huit experts de haute moralité et possédant une compétence reconnue dans le domaine visé par la Convention. Les États parties doivent périodiquement soumettre au Comité des rapports sur les mesures qu'ils ont adoptées pour donner effet à la Convention. Ce Comité, qui peut demander des renseignements supplémentaires aux États, soumet tous les deux ans un rapport sur ses activités à l'Assemblée générale des Nations Unies. Le Comité ne pouvant recevoir de communications individuelles ${ }^{46}$, le système de contrôle de la mise en œuvre de la Convention est donc essentiellement basé sur les rapports préparés par les États contractants euxmêmes et sur la publicité qui leur est donnée. Le Comité évalue ces documents et tente, par des recommandations, d'orienter le travail futur de l'État aux fins de respecter les exigences de la Convention. Ce mode de contrôle extrêmement respectueux de la souveraineté étatique a également été retenu par le Protocole facultatif: le non-respect de ce texte n'entraîne aucune sanction, les États parties devant seulement, dans les deux années qui suivent l'entrée en vigueur du Protocole, soumettre au Comité des droits de l'enfant un rapport en ce qui les concerne, puis un rapport quinquennal (art. 8 du Protocole facultatif).

La protection des enfants soldats n'est plus aujourd'hui limitée à cette stricte surveillance des dispositifs conventionnels, car elle n'a pas échappé à la «vogue» onusienne des procédures thématiques. Cette technique, qui apparaît au début des années 1980, consiste à désigner un expert indépendant, qui est chargé de surveiller soit un État, soit un aspect spécifique des droits de l'homme internationalement protégés ${ }^{47}$.

Dans son étude fondatrice de 1996, Graça Machel invitait le système des Nations Unies à examiner les questions relatives aux enfants touchés par les conflits armés de manière plus approfondie. Le rapport présentait un certain nombre de recommandations précises qui appelaient des changements dans l'action menée par le système des Nations Unies en faveur de la protection des enfants dans le contexte de conflits armés. Ces recommandations

44. Ibid., p. 522 et 633 .

45. Même si certaines institutions onusiennes militent pour une définition beaucoup plus globalisante de la notion de "participation directe». Voir notamment le Guide du Protocole facultatif concernant l'implication des enfants dans les conflits armés, UNICEF / Coalition contre l'utilisation des enfants soldats, mai 2004, p. 14.

46. À la différence d'autres instances comparables, telles que le Comité des droits de l’homme, le Comité pour l'élimination de la discrimination raciale, ou encore le Comité contre la torture.

47. Voir notamment O. de Frouville, Les Procédures thématiques: une contribution efficace des Nations Unies à la protection des droits de l'homme, Paris, Pedone, 1996. 
ont abouti dès 1996 à la désignation d'un représentant spécial du Secrétaire général pour les enfants et les conflits armés ${ }^{48}$. Souvent présenté comme un «porte-parole» des enfants touchés par la guerre, il diffuse ses recommandations dans un rapport annuel. Son rôle consiste essentiellement en une mission de sensibilisation et de mobilisation, à promouvoir l'application des normes internationales qui régissent la protection des enfants dans les conflits armés, proposer des initiatives qui amènent les parties au conflit à prendre des engagements spécifiques en vue de protéger les enfants et faire de la protection des enfants une priorité dans le cadre des processus et des opérations de paix. Le représentant spécial permet, en outre, d'améliorer la collaboration entre les organismes compétents des Nations Unies, les institutions spécialisées et autres instances concernées ainsi que les organisations non gouvernementales, qui agissent en matière de protection des enfants dans les conflits armés. Instrument désormais important de la «diplomatie humanitaire » menée par les Nations Unies, il recherche, par ses visites sur le terrain ${ }^{49}$, à obtenir des belligérants (qu'il s'agisse de groupes gouvernementaux ou de groupes rebelles) qu'ils prennent des engagements ou qu'ils adoptent des mesures de rétablissement après les conflits pour assurer la protection des enfants et le respect de leurs droits dans ces circonstances $^{50}$.

\section{B. L'apparition progressive d'institutions de coercition et de répression}

La volonté explicite de renforcer les pressions internationales sur les États concernés par le phénomène des enfants soldats a tout d'abord entraîné un engagement progressif du Conseil de sécurité des Nations Unies.

Organe principal des Nations Unies, le Conseil de sécurité possède la responsabilité principale du maintien de la paix et de la sécurité internationale. Il n'est donc pas, a priori, directement concerné par la protection des droits de l'homme, dans l'esprit de la Charte des Nations Unies $^{51}$. Mais le Conseil a progressivement acquis une place importante dans la protection des droits de l'homme au plan international. Depuis la fin des années 1990, il a notamment accordé une place de plus en plus grande à la question des enfants soldats et a condamné fermement les violations des règles internationales interdisant le recrutement et l'utilisation des enfants dans les conflits armés ${ }^{52}$. Le Conseil de sécurité a visé sans distinction toute partie à des conflits armés : non seulement les États, mais aussi les acteurs non étatiques, tels que les groupes armés engagés dans un conflit armé interne. Dès août 1999, le Conseil de sécurité des Nations Unies «condamne énergiquement» le recrutement et l'utilisation d'enfants dans les conflits armés en violation du droit international, et enjoint à toutes les parties concernées de mettre fin à de telles pratiques ${ }^{53}$. De manière encore plus explicite, en août 2000, le Conseil de sécurité estime que les violations systématiques, flagrantes et généralisées du droit international humanitaire et du droit relatif aux droits de l'homme, $y$ compris aux droits de l'enfant, dans les situations de conflit armé «peuvent constituer une menace contre la paix et la sécurité internationales ${ }^{54}$. Cette qualification permettrait donc en théorie au Conseil de sécurité de recourir aux mesures prévues à l'article 41 de la Charte des Nations Unies en tant que mesures coercitives pour garantir l'application du droit qui protège les enfants. Même si ces techniques n'ont jusqu'à présent jamais été utilisées, le Conseil de sécurité n'a pas cessé de peser sur les États confrontés à l'utilisation des enfants soldats. En effet, à partir de novembre 2001, il prie le Secrétaire général de lui présenter une liste des parties à des conflits armés qui recrutent ou utilisent des enfants dans des situations dont le Conseil est saisi (mécanisme de la « liste d'infamie» ou «naming and shaming» ${ }^{55}$. Le Conseil évoque également pour la première fois l'idée de sanctions et la nécessité d'intégrer les enfants dans des programmes dits «DDR» (désarmement, démobilisation, réintégration ${ }^{56}$. La présentation de cette liste, annexée au rapport annuel du Secrétaire général des Nations Unies sur les enfants et les conflits armés, permet au Conseil de sécurité d'entamer un dialogue avec les parties à des conflits armés, qui recrutent et utilisent des enfants, en vue d'élaborer des plans d'action «clairs et assortis d'échéances" pour mettre fin à cette pratique ${ }^{57}$. Les États et les groupes armés mentionnés doivent donner des informations sur les mesures prises pour mettre fin au recrutement et à l'utilisation d'enfants. Si le Conseil de sécurité estime ensuite insuffisants les progrès accomplis, il envisagera « de prendre des mesures appropriées pour résoudre ce problème, conformément à

48. Résolution 51 / 77 de l'Assemblée générale des Nations Unies, 12 décembre 1996. L’Assemblée générale a prorogé depuis le mandat du représentant spécial, dont le plus récemment par la nomination de R. Coomaraswamy en mars 2006.

49. 17 États sont concernés, à des titres divers, par des actions sur le terrain du représentant spécial en 2006. Voir http://www.un.org/french/special-rep/ children/index.html.

50. Voir notamment «Protection des enfants touchés par les conflits armés », note du Secrétaire général, doc. A / 55/442, 3 octobre 2000.

51. À la différence de l'Assemblée générale ou du Conseil économique et social. Voir les articles 13 et 62 de la Charte de San Francisco.

52. Voir N. Arzoumanian et F. Pizzutelli, «Victimes et bourreaux : questions de responsabilité liées à la problématique des enfants-soldats en Afrique ", Revue internationale de la Croix-Rouge, vol. 85, $\mathrm{n}^{\circ} 852$, décembre 2003, p. 827 sq.

53. Résolution 1261 (1999), Doc. S / RES / 1261 (1999), \$2.

54. Résolution 1314 (2000), Doc. S / RES / 1314 (2000), \$9.

55. La résolution 1460 en octobre 2003 présentait une liste incluant 54 forces dans 15 États, présentant notamment les États les plus confrontés au recrutement d'enfants, tels que la Colombie, le Myanmar et l'Ouganda. Par sa résolution 1539 d'avril 2004, le Conseil de sécurité a demandé à certaines forces désignées en Afghanistan, au Burundi, en Côte d'Ivoire, en République démocratique du Congo, au Liberia et en Somalie de développer des plans d'action concrets pour mettre fin au recrutement d'enfants.

56. Résolution 1379 (2001), doc. S / RES / 1379 (2001).

57. Voir notamment la Résolution 1460 (2003), UN Doc. S / RES / 1460 (2003), \$ 4. 
la Charte des Nations Unies et à sa résolution 1379 (2001) ». La résolution 1539, adoptée en 2004, prévoit un dispositif « gradué » pour les situations à l'ordre du jour du Conseil - élaboration de plans d'action pour mettre fin aux recrutements, qui peuvent même conduire à des sanctions en cas d'inaction ${ }^{58}$. Elle autorise également l'élargissement des listes d'infamie à d'autres violations des droits de l'enfant que le seul recrutement d'enfants soldats. Enfin, elle demande au Secrétaire général de l'organisation une réforme du mécanisme de contrôle et de rapport des Nations unies - dit «monitoring and reporting» - permettant au Conseil de disposer rapidement d'informations fiables et précises sur les recrutements et autres violations graves. Enfin, la résolution 1612 , adoptée en 2005 , met en place un mécanisme de suivi et d'évaluation, permettant une remontée plus efficace et mieux coordonnée de l'information jusqu'au Conseil, en commençant par cinq situations inscrites à l'ordre du jour du Conseil : République démocratique du Congo, Burundi, Côte d'Ivoire, Soudan et Somalie. Cette résolution prévoit également la création d'un groupe de travail du Conseil, qui s'est réuni pour la première fois le 17 novembre 2005, à New York, et qui a notamment pour mission de formuler des recommandations au Conseil de sécurité.

Enfin, il ne faut pas oublier que le renforcement de la pression sur les États de la communauté internationale passe également (et probablement encore davantage) par le développement de la répression ${ }^{59}$. L'avènement de la Cour pénale internationale constitue à cet égard un événement de grande importance. Le Statut de Rome de la Cour pénale internationale, adopté le 17 juillet $1998^{60}$, considère que le fait de procéder «à la conscription ou à l'enrôlement d'enfants de moins de 15 ans dans les forces armées nationales ou de les faire participer activement à des hostilités » constitue un crime de guerre, car relevant des infractions graves aux Conventions de Genève du 12 août $1949{ }^{61}$. Cette notion de " participation active », plus large que celle de "participation directe» généralement retenue en droit humanitaire, doit s'entendre à la fois dans le sens de participation directe au combat et dans celui de participation effective à des activités en rapport avec le combat, telles que la reconnaissance, l'espionnage, le sabotage, tout comme l'utilisation d'enfants comme leurres, messagers ou aux postes de contrôle militaires ${ }^{62}$. Les tra- vaux préparatoires du Statut de Rome ont d'ailleurs volontairement choisi d'interpréter de manière extensive la participation des enfants aux hostilités ${ }^{63}$. Cette disposition constitue indéniablement l'une des mesures les plus novatrices du Statut ${ }^{64}$. Les organisations non gouvernementales n'ont toutefois pas réussi à imposer un âge limite de 18 ans pour toute conscription et ont dû se plier à la volonté des États (dont celle des États-Unis) qui souhaitaient l'inscription d'un seuil de 15 ans.

Les premières affaires portées à la connaissance de la Cour montrent que la question des enfants soldats est effectivement prise en compte par cette juridiction. Au mois de décembre 2003, le président ougandais Yoweri Museveni a pris la décision de déférer la situation concernant l'Armée de résistance du seigneur au procureur de la Cour pénale internationale, qui a déterminé qu'il existait une base suffisante pour commencer à préparer la première enquête de la Cour. La situation en Ouganda porte sur des accusations d'enlèvements - principalement d'enfants - de meurtres, de tortures et de violences sexuelles à grande échelle. De même, pour la République démocratique du Congo, où la situation renvoyée au Bureau du procureur fait état de milliers de victimes tuées lors de massacres ou d'exécutions sommaires depuis 2002. Il est également question de viols et d'actes de torture commis à grande échelle, sans oublier l'utilisation massive d'enfants soldats ${ }^{65}$. La même approche a été retenue par la Cour spéciale pour le Liberia, qui a estimé que les questions de recrutement forcé des enfants relevaient de sa compétence. Parmi les inculpés devant cette juridiction pénale, figure notamment l'ancien président libérien Charles Taylor, accusé entre autres crimes d'avoir recruté des enfants de moins de 15 ans parmi ses milices armées ${ }^{66}$.

La protection internationale des enfants soldats offre donc le spectacle d'une accumulation de normes, de structures et d'acteurs agissant de manière conjointe. Malgré cette indéniable mobilisation, on doit constater que le bilan en la matière n'est que partiel et laisse en suspens de nombreuses questions concrètes sur le terrain.

\section{Un bilan en " demi-teinte »}

Le droit ne protège que dans la mesure où il est respecté et appliqué. Or si le droit international - et tout

58. Pouvant notamment consister en l'imposition de restrictions au déplacement des dirigeants, l'exclusion de ces derniers de toutes les structures de gouvernance et de toutes les dispositions d'amnistie, l'interdiction de l'exportation ou de la vente d'armes légères, l'interdiction de toute aide militaire, la restriction de l'apport de ressources financières aux parties en infraction et l'interdiction du commerce illicite des ressources naturelles. Voir Rapport du Secrétaire général sur les enfants et les conflits armés, doc A / 59/695-S/2005/72, 9 février 2005, \$115.

59. Dans sa résolution 1460 (2003), le Conseil de sécurité insiste «sur la responsabilité qu'ont tous les États de mettre fin à l'impunité et de poursuivre les auteurs de génocide, de crimes contre l'humanité, de crimes de guerre et d'autres crimes abominables commis contre des enfants ".

60. Et entré en vigueur le $1^{\text {er }}$ juillet 2002. Au 15 juin 2006, il lie 100 États parties.

61. Art. 8.2 a) xxvi).

62. Promotion et protection des droits de l'enfant; Assemblée générale des Nations Unies, $53^{\mathrm{e}}$ Session, $3{ }^{\mathrm{e}}$ Commission, point 106 de l'ordre du jour, Déclaration du CICR, New York, 21 octobre 1998.

63. Voir le commentaire du paragraphe 2) a xxvi) de l'article 8 du Statut de Rome de la Cour pénale internationale que donne C. Garraway dans The International Criminal Court: Elements of crimes and rules of procedure and evidence, R. S. Lee et al. (dir.), Londres, Transitional Publishers, $2001, \mathrm{p} .205$.

64. Et elle a d'ailleurs accéléré l'adoption du Protocole facultatif à la Convention sur les droits de l'enfant en 200o. Ce texte, en effet, «prend acte » de l'adoption du Statut de Rome dans son Préambule.

65. Voir Doc. A / 60 / 177, $1^{\text {er }}$ août 2005, Rapport de la Cour pénale internationale à l'Assemblée générale des Nations Unies.

66. Source: http://news.amnesty.org/index/ENGAFR514062004. 
spécialement son volet humanitaire - contient des dispositions d'une portée considérable pour protéger les enfants en temps de guerre, l'effectivité de la protection s'avère largement sujette à caution, ce droit restant peu efficient (A). La communauté internationale se heurte, en outre, à la question difficile du retour à la vie civile des enfants soldats (B).

\section{A. Un droit protecteur mais insuffisamment efficient}

Le droit international - et tout spécialement son volet humanitaire - contient des dispositions d'une portée considérable pour protéger les enfants en temps de guerre, mais l'effectivité de la protection s'avère largement sujette à caution. Il n'existe ni « police humanitaire », ni « forces armées humanitaires » qui seraient chargées de faire respecter ce droit. Cette responsabilité incombe tout d'abord aux États ${ }^{67}$, puis à la communauté internationale. Les Nations Unies ne se voient d'ailleurs que comme un niveau complémentaire et rappellent très régulièrement que "c'est aux gouvernements qu'incombe au premier chef la responsabilité officielle, juridique et politique d'assurer la protection de tous les enfants exposés à un conflit armé au sein de leur pays [...] les autorités nationales [ayant] un rôle central et direct à jouer pour assurer la protection effective de tous les enfants en danger et leur porter secours ${ }^{68}$. Si l'on se limite à l'application du droit humanitaire, les États parties aux Conventions de Genève de 1949 et aux Protocoles additionnels de 1977 se sont spécialement engagés à respecter et à faire respecter ce droit. Mais la faiblesse des moyens et techniques de contrôle est patente et limite largement la portée de la protection normative apportée aux enfants soldats. Comme l'ont indiqué certains auteurs, le droit international des conflits armés « est probablement la branche la moins respectée, et par conséquent aussi la plus théorique, sinon la plus utopique du droit international et même du droit tout court ${ }^{69}$. Les dispositions du droit international actuellement en vigueur pourraient suffire, pour l'essentiel, à faire cesser les violations les plus manifestes des droits de l'enfant lors des conflits armés. Or les moyens d'appliquer ce droit font encore largement défaut ${ }^{70}$.

La Cour pénale internationale n'en est qu'à ses balbutiements, même si la ratification du Statut de Rome par de nombreux États africains est de nature à envisager le développement de sanctions dans le cadre de conflits impliquant le recours à des enfants soldats. Il n'en reste pas moins que parmi les cinq États inscrits à l'ordre du jour du Conseil de sécurité depuis 2005 (voir ci-dessus), seuls la République démocratique du Congo et le Burundi ont ratifié le statut, ce qui n'est pas le cas de la Côte d'Ivoire, du Soudan et de la Somalie. Souvent évoqués dans les documents onusiens ${ }^{71}$, le Myanmar et le Népal ne sont pas davantage parties au statut. L'intervention de sanctions au niveau international pour réprimer le recours aux enfants soldats est donc, au mieux, en devenir ${ }^{72}$. La même constatation peut être faite concernant l'intervention du Conseil de sécurité qui, s'il s'est doté depuis sa résolution 1539 (2004) de mécanismes d'action gradués pour mettre fin aux recrutements d'enfants soldats, pouvant aller jusqu'à de véritables sanctions à l'encontre des États concernés, il n'a jamais, à ce jour, utilisé ces possibilités. Les problèmes de fonctionnement du Conseil et les difficultés récurrentes pour trouver une majorité susceptible d'adopter les résolutions, tout en évitant un veto de l'un des cinq membres permanents, ne laissent guère espérer une forte utilisation de ces procédures de sanction.

Si les moyens d'action du Conseil de sécurité et de la Cour pénale internationale sont "en devenir », ceci n'est normalement pas le cas pour l'application du droit conventionnel de protection des enfants soldats. Les normes sont aujourd'hui connues et entrées en vigueur depuis de nombreuses années (à l'exception notable, toutefois, du Protocole facultatif, entré en vigueur en 2002 seulement) et ont fait l'objet d'un nombre de ratifications que l'on peut estimer comme très satisfaisant. Chacun sait toutefois que la ratification d'une convention internationale ne signifie pas ipso facto son application et son respect par l'État partie. Ainsi la Sierra Leone fut l'un des premiers États à ratifier la Convention sur les droits de l'enfant de 1989 (ratification du 7 septembre 1990) et le Protocole facultatif de 2000 (ratification du 15 mai 2002), mais les dispositions de ces deux textes sont largement restées lettre morte en droit interne. La même remarque peut être faite en ce qui concerne la République démocratique du Congo, partie à la Convention relative aux droits de l'enfant depuis le 27 septembre 1990 et au Protocole facultatif depuis le 11 novembre $2001^{73}$... Ces exemples pourraient être multipliés et sont largement dus à la faiblesse

67. Voir, en particulier, l'article 146 de la IV Convention de Genève et les articles 85 et 86 du Protocole I.

68. Doc. A/59/695-S/ $2005 / 72, \$ 108$

69. E. David, Principes de droit des conflits armés, Bruxelles, Bruylant, 1994, p. 522.

70. Si ce n'est même la simple connaissance de ces dispositions... À ce titre, les campagnes de sensibilisation à l'intention du public, de l'armée et des autorités sont également importantes, car une norme qui n'est pas connue ne peut être efficace.

71. Pour une liste récente des États concernés à des titres divers par la question des enfants soldats, voir les annexes I et II du doc. A / 59 / $695-\mathrm{S} / 2005 / 72$.

72. Il ne faut toutefois pas oublier qu'en vertu de l'article 25 du Statut, la Cour pénale internationale peut exercer sa compétence à l'égard d'États sur le territoire duquel le crime a été commis, ou d'États dont la personne accusée du crime est un ressortissant. En outre, un État qui n'est pas partie au Statut peut, par déclaration, consentir à ce que la Cour exerce sa compétence. Enfin, dans le cadre du régime de sécurité collective prévu au chapitre VII de la Charte des Nations Unies, le Conseil de sécurité peut déférer une situation au procureur pour enquête.

73. On peut même souligner que l'article 184 de la Constitution de transition, adoptée à Sun City le $1^{\text {er }}$ avril 2003, prévoit que nul ne peut être recruté dans les forces armées de la République démocratique du Congo ni prendre part à des guerres ou à des hostilités s'il n'a pas atteint l'âge de 18 ans révolus au moment du recrutement. En outre, en vertu du décret-loi nº 066 du 9 juin 2000 portant démobilisation et réinsertion des groupes vulnérables présents au sein des forces combattantes, un ordre de démobilisation et de réinsertion familiale et/ ou socio-économique des groupes vulnérables, présents au sein des forces armées congolaises ou dans tout autre groupe armé, est lancé sur l'ensemble du territoire de la République démocratique du Congo (art. $1^{\text {er }}$ ). 
des moyens internationaux de contrôle de l'application des traités.

Les résultats du contrôle effectué par le Comité des droits de l'enfant, chargé tout à la fois de la surveillance de l'application de la Convention de 1989, comme du Protocole facultatif de 2000, apparaissent d'une intensité particulièrement réduite par rapport à l'étendue de la question. Ainsi lorsqu'en 1999, il juge préoccupant le non-respect des droits des enfants dans les régions de l'État partie en proie à des conflits armés, comme la Tchétchénie et le Daghestan, et s'inquiète, en particulier, de la participation d'enfants aux conflits armés, il se contente d'encourager «l'État partie à veiller à ce que les enfants et autres civils soient protégés en période de conflit et à ce qu'un soutien et une aide à la réadaptation, notamment une assistance psychologique, soient fournis aux enfants déplacés à l'intérieur du pays et aux enfants vivant dans des régions touchées par des conflits armés ${ }^{74}$. Lors de l'examen du rapport de la Papouasie-Nouvelle-Guinée en 2004, le Comité des droits de l'enfant estime que « tout en reconnaissant les efforts accomplis par l'État partie en vue de la réinsertion des anciens enfants soldats, le Comité n'en est pas moins préoccupé de voir qu'un nombre important d'enfants continue de subir les conséquences très néfastes du conflit armé ». Mais les moyens de l'organe de contrôle apparaissent alors assez dérisoires, le Comité se contentant de recommander «à l'État partie de mettre en place une stratégie globale visant à veiller à ce qu'aucun enfant ne soit impliqué dans le conflit armé et à ce que tout ancien enfant soldat fasse l'objet de mesures adéquates de réadaptation et de réinsertion dans la société ${ }^{75}$. La même constatation peut être faite à l'encontre du Soudan, où le Comité, après avoir mentionné que les enfants soldats continuent à être employés par le Gouvernement et les forces d'opposition, se contente de recommander à l'État partie et, dans la mesure du possible, aux autres acteurs concernés, «la fin du recrutement et de l'utilisation d'enfants soldats, conformément aux règles internationales applicables ${ }^{76}$. Le Comité apparaît d'ailleurs totalement dépassé face à des situations de violations massives des droits des enfants, comme le montre bien l'exemple de la Sierra Leone. Face à un État présentant un «nombre très élevé d'enfants qui ont été recrutés de force dans les forces armées, y compris dès l'âge de 5 ans, et qui ont souvent été contraints à commettre des atrocités envers d'autres personnes, notamment envers d'autres enfants et membres de leur communauté», le Comité ne peut que «prier instamment l'État partie de prendre toutes les mesures possibles pour que tous les enfants enlevés et les enfants soldats soient libérés et démobilisés et pour qu'ils soient réadaptés et réinsérés dans la société » et recommander à cet État l'adoption d'une loi interdisant à l'avenir le recrutement des enfants de moins de 18 ans ${ }^{77}$. La portée du contrôle de l'application du Protocole facultatif par le Comité des droits de l'enfant apparaît impossible à apprécier pour l'instant, les rapports étatiques examinés jusqu'à présent par le Comité ne relevant pas réellement d'États parties concernés par l'utilisation des enfants soldats ${ }^{78}$.

La faiblesse des moyens d'action internationaux s'avère tout à fait marquante en ce qui concerne les perspectives d'accompagnement du retour à la vie civile des enfants soldats : l'action internationale est présente, mais les résultats restent encore trop limités et souvent fragiles.

\section{B. Un difficile accompagnement du retour à la vie civile des enfants soldats}

En vertu de l'article $6 \$ 3$ du Protocole facultatif de 2000, «Les États parties prennent toutes les mesures possibles dans la pratique pour veiller à ce que les personnes relevant de leur compétence qui sont enrôlées ou utilisées dans des hostilités en violation du présent Protocole soient démobilisées ou de quelque autre manière libérées des obligations militaires. Si nécessaire, les États parties accordent à ces personnes toute l'assistance appropriée en vue de leur réadaptation physique et psychologique et de leur réinsertion sociale ». Dans sa résolution 1379 du 20 novembre 2001, le Conseil de sécurité a en outre explicitement demandé aux États parties à des conflits armés de "prévoir la protection des enfants dans les accords de paix, $y$ compris, le cas échéant, des mesures de désarmement, de démobilisation, de réinsertion et de rééducation des enfants soldats et de regroupement des familles, en tenant compte, lorsque cela est possible, de l'avis des enfants ».

Cette prise en compte du retour à la vie civile pour les enfants soldats n'est pas seulement guidée par des objectifs humanitaires et le respect du bien-être des enfants démobilisés. La participation d'enfants aux hostilités peut, en effet, avoir une influence néfaste à long terme sur la société. En effet, les enfants, victimes de la guerre et laissés à l'abandon, deviennent vite des $"$ créateurs de guerre ${ }^{79}$, car, séparés de leur famille, ils représentent un risque extrême d'être recrutés ou réengagés au sein de groupes armés ${ }^{80}$.

74. Doc. CRC / C / 90, 7 décembre 1999, \$118.

75. Comité des droits de l'enfant, $35^{\mathrm{e}}$ session, 12-30 janvier 2004, Observations finales: Papouasie-Nouvelle-Guinée, $\$ 518$ et 519.

76. Comité des droits de l'enfant, Concluding observations : the Sudan, doc. CRC / C / 15 / Add.190, 9 octobre 2002, \$59- 60.

77. Comité des droits de l'enfant, Observations finales : Sierra Leone, doc. CRC / C / 15/ Add.116, 24 février 2000, $\$ 71$ et 73.

78. En mars 2006, les observations du Comité des droits de l'homme avaient seulement concerné les rapports étatiques d'Andorre, du Bangladesh, de la Suisse, du Danemark et de la Finlande.

79. Voir la résolution $1460(2003)$ du 30 janvier 2003 dans laquelle le Conseil de sécurité « engage les États membres et les organisations internationales à veiller à ce que les enfants touchés par les conflits armés soient associés à tous les processus de désarmement, de démobilisation et de réinsertion [...] en mettant particulièrement l'accent sur l'éducation et le suivi des enfants démobilisés, notamment dans les écoles, pour empêcher qu'ils ne soient de nouveau recrutés» (souligné par nous).

80. Ainsi en Côte d'Ivoire, les forces armées continuent de recruter des jeunes Libériens dans les camps de réfugiés de l'ouest du pays. Source: Rapport du Secrétaire général sur les enfants et les conflits armés, doc. S/ 2003/1053, S. De même, au Tchad, les camps de réfugiés du Darfour sont devenus un vivier pour la rébellion soudanaise, qui y recrute des combattants âgés parfois de 14 ans (Le Monde, 30 juin 2006). 
D'où une volonté de faire pression sur les différentes factions pour qu'elles relâchent les enfants, ceux-ci étant ensuite pris en charge par différentes structures aux fins de les soigner psychiquement, de les éduquer et de les réinsérer.

Le représentant spécial des Nations Unies, assisté de nombreuses institutions onusiennes, a œuvré depuis 1997 en faveur d'accords avec les forces sur le terrain tendant à protéger les enfants et à œuvrer pour leur libération. Ces efforts ne sont pas totalement restés lettre morte et certains succès sont indéniables. Au Liberia, lors de la visite de juin 1998, le Groupe de contrôle de la Communauté économique des États de l'Afrique de l'Ouest (ECOMOG) et les Forces de défense civile sont convenus de fournir une protection spéciale aux enfants combattants du Conseil révolutionnaire des forces armées / Front révolutionnaire uni (CRFA / FRU) qui, après s'être rendus, avoir été capturés ou avoir fui, se retrouveraient sous leur garde. Le FRU s'est, en outre, engagé à ne pas recruter d'enfants âgés de moins de 18 ans. Au Sri Lanka, en 1998, les Liberation Tigers of Tamil Eelan (LTTE) se sont engagés à ne pas utiliser d'enfants de moins de 18 ans dans les combats et à ne pas enrôler d'enfants de moins de 17 ans. En Colombie, au cours de la visite du représentant spécial en juin 1999, le président a annoncé qu’il mettrait fin immédiatement à l'enrôlement dans l'armée de jeunes de moins de 18 ans et qu'il renverrait dans leurs foyers tous ceux qui s'y trouvaient. À la fin de l'année, tous les soldats âgés de moins de 18 ans avaient été démobilisés. Toujours en 1999, le représentant spécial a obtenu du Revolutionary United Front (RUF) sierra leonais qu'il s'engage à libérer les enfants qu'il avait enlevés et les enfants soldats, tandis que les Forces de défense civiles se sont engagées à ne pas recruter d'enfants soldats et à démobiliser ceux qui avaient été enrôlés. L'exécution de ces engagements a pu être suivie par la MINUSIL et l'UNICEF. En République démocratique du Congo, le représentant spécial a soulevé auprès du RCD la question de l'enrôlement d'enfants dans les rangs du Rassemblement et celui-ci a accepté le principe de ne pas recruter de jeunes gens âgés de moins de 18 ans et a décidé que des mécanismes précis et des dispositions concrètes seraient mis en place à cette fin ${ }^{81}$. D'une manière plus générale, le représentant spécial pour les enfants dans les conflits armés soulignait en 2003 que quinze États parties ont pris une soixantaine d'engagements auprès de lui ${ }^{82}$.

En outre, les accords de paix contiennent effectivement de plus en plus des engagements à l'égard des enfants touchés par des conflits armés. On peut, à ce titre, citer notamment l'accord du Vendredi Saint conclu en Irlande du Nord (1998), l'accord de paix de Lomé sur la Sierra Leone $(1999)^{83}$, l'accord d'Arusha pour la paix et la réconciliation au Burundi (2000), qui contiennent des mentions concernant la protection des enfants et l'obligation de leur accorder la priorité dans les activités de consolidation de la paix et de reconstruction entreprises au lendemain de conflits. Plus récemment l'accord de cessez-le-feu conclu en février 2002 entre le gouvernement sri lankais et les Tigres tamouls (LTTE) a offert l'occasion d'améliorer la situation des enfants. De même en Angola, à la suite de la signature en avril 2002 d'un accord de cessez-le-feu, un vaste processus de démobilisation et de réinsertion des ex-combattants a été engagé. L'accord de paix signé à Accra le 18 août 2003 prévoit d'accorder une protection aux enfants touchés par la guerre au Liberia en vue de les réinsérer dans la société civile. Ces constatations positives ne doivent toutefois pas occulter le fait que de nombreux conflits n'intègrent toujours pas la protection des enfants dans les processus de paix ou de réconciliation nationale: au Cambodge, au Salvador, au Liberia, au Guatemala, au Mozambique notamment, aucune attention n'est portée aux enfants, ni stipulée dans l'accord de paix, ni prévue dans le processus de désarmement, de démobilisation et de réinsertion.

Si l'action internationale est aujourd'hui indéniable, la réalité sur le terrain se présente rarement comme prévu. Les acteurs locaux et internationaux n'ont pas toujours les ressources, les structures programmatiques et les connaissances nécessaires pour faire face aux démobilisations spontanées ou massives, pour remettre les ex-enfants soldats à leur famille, pour les réinsérer durablement dans la société, d'où des échecs tout à fait marquants. Comme l'indique le représentant spécial des Nations Unies « il est indispensable de suivre systématiquement la situation et d'exercer des pressions pour faire en sorte que les engagements pris soient tenus» ${ }^{84}$.

Plusieurs situations concrètes prouvent malheureusement la véracité de ce constat plutôt négatif. En Angola, quelque 8000 enfants recrutés pendant la guerre civile ont été rendus à la vie civile sans que leur libération intervienne dans le cadre d'un processus officiel de démobilisation. Au Liberia, malgré tous les efforts de l'UNICEF et des organisations non gouvernementales, la démobilisation hâtive des quelque 21000 combattants, dont 4300 enfants, entre novembre 1996 et février 1997, n'a pratiquement rien offert aux enfants soldats. Des milliers d'enfants soldats libériens n'ont pas été officiellement démobilisés et sont restés dans la brousse sous le contrôle de facto de

81. Action du représentant spécial qui s'est accompagnée d'une forte pression internationale. Le Conseil de sécurité des Nations Unies indique en 2003 qu'il « condamne avec force le fait que des enfants continuent à être recrutés et utilisés dans les hostilités en République démocratique du Congo, en particulier dans le Nord et le Sud-Kivu et dans l'Intru...» (Résolution 1493 (2003), doc. S / RES / 1493 (2003)). Quant à la Commission des droits de l'homme des Nations Unies, elle « demande instamment à toutes les parties de mettre fin au recrutement et à l'utilisation d'enfants soldats en violation du droit international... » et prie le gouvernement de fournir des informations sur les mesures prises pour faire respecter la législation applicable en matière de recrutement forcé ou obligatoire d'enfants dans les conflits armés (Résolution $\mathrm{n}^{\circ} 84$ du 22 avril 2004).

82. Doc. A / $58 / 328,29$ août $2003, \$ 22$.

83. L'UNICEF a été l'organe chef de file de la protection des enfants pendant ce processus, qui s'est déroulé entre 1998 et 2001 . Les enfants démobilisés ont été accueillis dans des centres de soins temporaires où ils ont reçu des soins de santé et un appui psychosocial et ont participé à des activités d'éducation et de loisirs pendant que l'on recherchait leurs parents aux fins du regroupement familial.

84. Doc. A/58/328, 29 août 2003, $\$ 22$. 
leurs chefs militaires. En République démocratique du Congo, à la mi-décembre 2004, près de 700 enfants avaient bénéficié du programme de démobilisation et un nombre indéterminé d'enfants avait été libéré par ces groupes avant le lancement dudit programme ${ }^{85}$. Toutefois la rapporteuse spéciale des Nations Unies sur la situation des droits de l'homme en République démocratique du Congo indiquait également que le phénomène des enfants soldats reste très préoccupant : la démobilisation est très réduite et le recrutement reste massif à l'est du pays (concernant plus de 30000 enfants soldats) ${ }^{86}$. En 2005 le Secrétaire général des Nations Unies se félicite qu' «aucun cas de recrutement d'enfants dans l'Armée nationale afghane n'a été signalé » et que la mise en œuvre du programme «Nouveau départ pour l'Afghanistan ${ }^{87}$ a permis la démobilisation de plus de 3820 enfants en 2004, mais ajoute immédiatement que « l'équipe de pays des Nations Unies n'a pas été en mesure d'identifier précisément les factions armées qui continuaient d'enrôler des enfants au cours de la période considérée » (soit de novembre 2003 à décembre 2004) et qu' " aucun engagement de mettre fin à cette pratique n'a été pris par ces factions ${ }^{88}$.

En outre certains groupes particuliers parmi les enfants soldats posent des problèmes particuliers : la démobilisation et le retour à la vie civile des jeunes filles s'avèrent en effet particulièrement difficiles. Le désarmement mis en place au Sierra Leone à partir de juillet 1999 n'a ainsi pas su toucher les filles qui avaient été enlevées et contraintes de fournir des services sexuels à des groupes armés. Étroitement surveillées par les chefs qu'elles devaient suivre, elles avaient trop peur pour revendiquer leur place dans le processus de démobilisation ${ }^{89}$. De même, les succès spectaculaires obtenus au Liberia, où en octobre 2004 plus de 10000 enfants - dont plus de 2300 filles - avaient été désarmés et démobilisés et plus de 9600 avaient rejoint leur famille, sont également à fortement tempérer en ce qui concerne les jeunes filles, car « environ $75 \%$ des filles démobilisées qui ont été hébergées dans les centres d'accueil provisoires auraient été victimes d'une forme ou d'une autre de violence et d'exploitation sexuelles ${ }^{90}$.

Le Secrétaire général sur les enfants et les conflits armés a parfaitement synthétisé les difficultés de l'action internationale en matière de protection des enfants soldats dans son rapport rendu public en 2005: « La communauté internationale fait actuellement face à une cruelle dichotomie. D'un côté, des normes clairement définies et rigoureuses ainsi que d'importantes initiatives concrètes, en particulier à l'échelon international, ont été mises au point pour assurer la protection des enfants dans les conflits armés. D’un autre côté, les atrocités dont sont victimes les enfants et l'impunité dont jouissent leurs auteurs se poursuivent quasiment sans relâche sur le terrain ${ }^{91}$. L'action internationale se heurte en effet non seulement au verrou traditionnel de la souveraineté étatique, mais aussi à la difficulté de faire accepter des principes humanitaires par des acteurs locaux qui ne respectent guère les règles d'une manière générale. Le droit peine ici à répondre aux difficultés posées par un problème aux causes multiples. Si l'action conjuguée du Conseil de sécurité et de la Cour pénale internationale constituera très probablement un élément dissuasif dans l'avenir, cela ne suffira certainement pas à éradiquer les causes des conflits. Extrême pauvreté, pillage des ressources naturelles, conflits ethniques et cynisme des trafiquants d'armes ne semblent pas, en effet, en voie de disparition. L'enfant, par sa double caractéristique de victime et d'acteur, risque malheureusement de demeurer l'une des figures marquantes des guerres du XXI ${ }^{\mathrm{e}}$ siècle.

85. Commission d'experts pour l'application des conventions et recommandations (CEACR): Observation individuelle concernant la Convention $\left(\mathrm{n}^{\circ} 182\right)$ sur les pires formes de travail des enfants, 1999, République démocratique du Congo (ratification : 2001$)$, Publication : 2006.

86. Doc. E / CN.4/ $2003 / 43, \S 33$ à 36.

87. Processus national de désarmement, de démobilisation et de réinsertion lancé en février 2004 et placé sous la conduite de l'UNICEF. Il a été mis en place dans le nord-est, l'est et les hauts plateaux du centre du pays.

88. Doc. A / 59 / 695-S / 2005/ 72, 9 février 2005, Rapport du Secrétaire général sur les enfants et les conflits armés.

89. Source : Guide du Protocole facultatif..., p. 19.

90. Rapport du Secrétaire général sur les enfants et les conflits armés, doc. A / 59/695-S/ 2005/ 72, 9 février 2005 , \$25 sq.

91. Doc A / 59 / 695-S/ 2005/ 72, 9 février 2005, \$ 61. 
